\title{
Trabectedin for advanced soft tissue sarcomas: optimizing use
}

\author{
This article was published in the following Dove Press journal: \\ Therapeutics and Clinical Risk Management \\ 12 December 2014 \\ Number of times this article has been viewed
}

\author{
Alison Reid \\ Juan Martin-Liberal \\ Charlotte Benson \\ Sarcoma Unit, The Royal Marsden \\ NHS Foundation Trust, London, UK
}

Correspondence: Alison Reid

The Royal Marsden NHS Foundation

Trust, Downs Road, Sutton,

Surrey SM2 5PT, UK

Tel +44208 66I 3458

Email alison.reid@rmh.nhs.uk
Abstract: Patients with locally advanced or metastatic soft tissue sarcoma have a poor outlook with median survival in the order of 1 year. There is therefore an urgent need for novel agents to impact this disease. Trabectedin is one such novel agent that has demonstrated activity for patients with advanced soft tissue sarcoma and it was licensed in Europe in 2007 for patients in the second-line setting or first-line in those patients deemed unsuitable to receive cytotoxics. In order to best serve patients with novel agents, it is imperative to understand the mechanism or mechanisms of action and the best ways of assessing response in order to optimize antitumor activity. Frequently, the mechanism of action and the optimal means of assessing response will be different from those of traditional cytotoxics. Trial design should reflect these factors to ensure that active drugs are not wrongly marked as futile. This review discusses a number of factors that may influence the optimization of trabectedin use. These factors include the administration schedule, the optimal timing of trabectedin administration in the disease process, the histopathological and molecular subtypes that may be most sensitive to trabectedin, the challenge of assessing response, particularly using radiology, and, finally, the safety considerations with this agent.

Keywords: trabectedin, soft tissue sarcomas, ET-743, myxoid lipoarcomas, optimal administration

\section{Introduction}

Soft tissue sarcomas are a relatively rare and heterogeneous group of tumors with at least 50 different histological subtypes. The outlook for patients with an unresectable or metastatic soft tissue sarcoma is poor, with a median overall survival in the order of 1 year. ${ }^{1}$ Despite the heterogeneity of soft tissue sarcomas, to date they have largely been considered collectively for clinical trial purposes. In patients with advanced/ metastatic soft tissue sarcoma, standard first-line chemotherapy involves a singleagent anthracycline (commonly doxorubicin). An anthracycline-based doublet (ie, ifosfamide and doxorubicin) may be appropriate for selected patients depending on histological subtype, fitness, and whether surgery may be possible if chemotherapyinduced downstaging is achieved. Response rates, however, are realized only in $20 \%-30 \%$ of patients ${ }^{2-4}$ and there is therefore a clear unmet need for novel therapeutics to impact this disease. One such agent demonstrating meaningful activity is trabectedin (ecteinascidin-743 or ET-743, Yondelis ${ }^{\circledR}$ ). Based on evidence of activity in the advanced setting, trabectedin was approved by the European Medicines Agency (EMA) in 2007 for the treatment of patients with advanced soft tissue sarcoma (ASTS) following first-line chemotherapy or as first-line therapy for those patients deemed unsuitable for cytotoxic treatment. Trabectedin has also been granted an orphan drug status by the Food and Drug Administration in the United States. This review examines 
the following issues that may influence the optimization of trabectedin's use:

1. optimal administration schedule;

2. timing of administration in the sarcomatous disease process;

3. differential trabectedin activity related to soft tissue sarcoma subtype;

4. the challenge of assessing response to trabectedin;

5. safety considerations.

\section{Mechanism of action}

It is worth considering first the putative mechanism of action of this agent, as this directly relates to issues of optimization such as safety, how best to assess response, and in which sarcoma subtypes the drug has shown most promise. Trabectedin is a marine-derived compound isolated from the Caribbean tunicate Ecteinascidia turbinata. It is a tetrahydroisoquinolone alkaloid composed of three fused rings that bind the N2 position of guanine in the minor groove of DNA, inducing a DNA bending toward the major groove (Figure 1). Trabectedin modulates gene expression in a promoter-dependent and gene-dependent manner. This observation was initially made in relation to the promoters of heat-shock proteins and MDR1 genes but has now been extended to other promoters and seems to be cell-dependent. ${ }^{5-7}$ Trabectedin inhibits transcription by preventing the binding of transcription factors to DNA. ${ }^{8,9}$ Secondly, although the drug covalently binds to only a single strand of DNA, it functionally mimics an interstrand cross-linking lesion. The interaction with the second strand is via van der Waals forces and hydrogen bonds only. By this mechanism, transcription is blocked

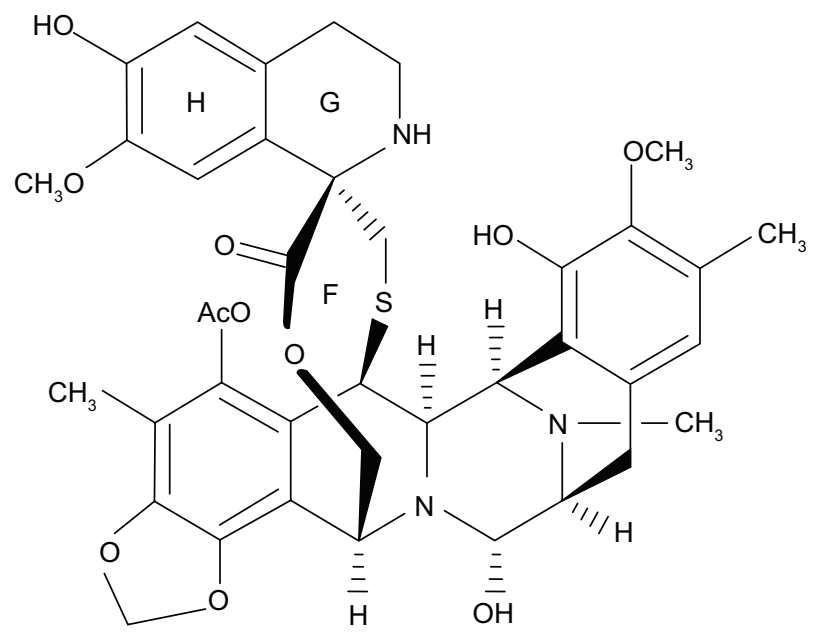

Figure I The chemical structure of trabectedin. through stabilization of double-stranded DNA (ds-DNA). ${ }^{7,10}$ Moreover, trabectedin interacts directly with the elongating RNA polymerase II as it attempts to catalyze the transcription of DNA to synthesize precursors of mRNA. ${ }^{7}$ Ultimately, this arrests RNA polymerase II during transcription elongation and results in its degradation via the proteasome pathway in a nucleotide excision repair way. ${ }^{11}$ While the principal mechanism of trabectedin's action seems to involve the interruption of transcription, there is also evidence that modulation of the production of cytokines and chemokines by tumor and normal cells can occur. ${ }^{12}$ The drug may therefore also be exerting an antitumor effect via the microenvironment. There are also some data in breast cancer cell lines to suggest that trabectedin causes activation of apoptotic pathways and produces reactive oxygen species. ${ }^{13}$ Additionally, there are some mechanisms of action that relate specifically to translocation-related sarcomas. These will be discussed in more detail in "Differential trabectedin activity related to soft tissue sarcoma subtype".

\section{Optimal administration schedule}

While the response rates reported from trials of trabectedin in ASTS were not particularly impressive, ranging from $4 \%$ to $8 \%$, it became apparent from the early trials that the drug was exerting a meaningful effect in terms of disease control with improved time to progression and progression-free survival (PFS) rates (Table 1). The majority of the early studies utilized a dose of $1.5 \mathrm{mg} / \mathrm{m}^{2}$ as a 24-hour infusion given every 3 weeks. This was confirmed to be superior to a once-weekly, 3-out-of-4 weeks schedule in a randomized Phase II trial comparing the traditional schedule of $1.5 \mathrm{mg} / \mathrm{m}^{2}$ 24-hour intravenous infusion every 3 weeks (q3w $24 \mathrm{~h}$ ) with trabectedin administered as a $0.58 \mathrm{mg} / \mathrm{m}^{2}$ 3-hour infusion once weekly for 3 weeks of a 4-week cycle (qw 3h). The primary end point was the time to progression (TTP) and the $\mathrm{q} 3 \mathrm{w} 24 \mathrm{~h}$ demonstrated a superior TTP of 3.7 months versus 2.3 months (hazard ratio [HR], 0.734; $95 \%$ confidence interval [CI], 0.554-0.974; $P=0.0302) .{ }^{14}$ The PFS rates observed in this study exceeded the European Organisation for Research and Treatment of Cancer (EORTC) criteria of an active agent in pretreated soft tissue sarcoma (these being PFS of at least $39 \%$ at 3 months and $14 \%$ at 6 months). ${ }^{15}$ Moreover, as trabectedin does not demonstrate the cumulative toxicity of a chemotherapeutic agent, approximately $20 \%$ of patients achieved long-term benefit receiving between 20 and 30 consecutive cycles. This trial was conducted in patients with liposarcoma and leiomyosarcoma who had received prior treatment with at least anthracycline and 
Table I Phase II studies of single-agent trabectedin in advanced soft tissue sarcomas

\begin{tabular}{|c|c|c|c|c|c|c|}
\hline Reference & $\begin{array}{l}\text { Line } \\
\text { of therapy }\end{array}$ & Pt no & Dose schedule & Response rate & 6-month PFS & $\begin{array}{l}\text { Median duration of } \\
\text { survival }\end{array}$ \\
\hline 14 & $\begin{array}{l}\text { Second } \\
\text { or third }\end{array}$ & 270 & $\begin{array}{l}\text { Randomized study - } \\
1.5 \mathrm{mg} / \mathrm{m}^{2} 24 \text {-hour infusion } \\
\mathrm{q} 3 \mathrm{w} \text { versus } 0.58 \mathrm{mg} / \mathrm{m}^{2} \\
3 \text {-hour infusion once weekly, } \\
3 \text { out of } 4 \text { wks }\end{array}$ & $\begin{array}{l}5.6 \%(24-\text { hour } \\
\text { infusion q3w) }\end{array}$ & $\begin{array}{l}35.5 \%(24-h o u r \\
\text { infusion } \mathrm{q} 3 \mathrm{w})\end{array}$ & $\begin{array}{l}\text { I } 3.9 \text { versus II } .8 \text { months } \\
\text { (HR } 0.843 ; 95 \% \mathrm{Cl} \text {, } \\
0.653-\mathrm{I} .090 ; P=0.1920) \\
\text { for } 24 \text { - versus 3-hour } \\
\text { schedule }\end{array}$ \\
\hline 25 & $\begin{array}{l}\text { Second, third, } \\
\text { and fourth }\end{array}$ & 36 & $\begin{array}{l}1.5 \mathrm{mg} / \mathrm{m}^{2} 24 \text {-hour infusion } \\
\mathrm{q} 3 \mathrm{w}\end{array}$ & $8 \%$ & $\begin{array}{l}\text { Not described. } \\
\text { Median TTP I.7 months } \\
\text { (95\% Cl, I.3-4.4 months) } \\
\text { I-year survival rate } 53 \%\end{array}$ & $\begin{array}{l}\text { I2.I months ( } 95 \% \mathrm{Cl} \\
8.1-26.5 \text { months) }\end{array}$ \\
\hline 26 & $\begin{array}{l}\text { Second } \\
\text { or third }\end{array}$ & 104 & $\begin{array}{l}1.5 \mathrm{mg} / \mathrm{m}^{2} 24 \text {-hour infusion } \\
\mathrm{q} 3 \mathrm{w}\end{array}$ & $8 \%$ & $29 \%$ & $\begin{array}{l}9.2 \text { months ( } 278 \text { days; } \\
95 \% \mathrm{Cl} 238-368)\end{array}$ \\
\hline 27 & First & 36 & $\begin{array}{l}1.5 \mathrm{mg} / \mathrm{m}^{2} 24 \text {-hour infusion } \\
\mathrm{q} 3 \mathrm{w}\end{array}$ & $17 \%$ & $24.4 \%(95 \% \mathrm{Cl}, \mathrm{I} 3-44)$ & Not documented \\
\hline 32 & $\begin{array}{l}\text { Second, third, } \\
\text { or fourth }\end{array}$ & 54 & $\begin{array}{l}1.5 \mathrm{mg} / \mathrm{m}^{2} 24 \text {-hour infusion } \\
\mathrm{q} 3 \mathrm{w}\end{array}$ & $4 \%$ & $24 \%$ & $\begin{array}{l}\text { I2.8 months (range, } \\
0.69-33.77 \text { months) }\end{array}$ \\
\hline
\end{tabular}

Abbreviations: PFS, progression free survival; TTP, time to progression; q3w, 3 weekly; pt no, patient number; HR, hazard ratio; Cl, confidence interval.

ifosfamide and resulted in the European approval of trabectedin administered on the 3-weekly schedule in ASTS second line (after failure of standard therapy with anthracyclines and ifosfamide) or first line in patients unsuited to receive anthracycline/ifosfamide.

\section{Timing of administration in the sarcomatous disease process}

\section{First-line setting}

Table 1 describes the early Phase II clinical trials with trabectedin in the second or subsequent line of treatment for ASTS and demonstrates the encouraging activity seen with this agent particularly in terms of PFS. Phase III trials in the advanced setting include an open-label, randomized (2:1) study of trabectedin versus dacarbazine in liposarcoma or leiomyosarcoma. ${ }^{16}$ The study is closed to accrual and the results are awaited. Given the activity demonstrated in the advanced setting (beyond first line), interest turned to examining trabectedin's activity in the first-line setting, both as a single agent and in combination. A number of trials are under way, have completed accrual, or have been reported. One study in the first-line setting has been published. This study is a randomized Phase III trial of first-line trabectedin versus doxorubicinbased chemotherapy and has been conducted in patients with translocation-related sarcoma subtypes. ${ }^{17}$ Patients were random$\operatorname{ized}(1: 1)$ to receive trabectedin $\left(1.5 \mathrm{mg} / \mathrm{m}^{2} 24 \mathrm{~h} \mathrm{q} 3 \mathrm{wk}\right)$, or doxorubicin $75 \mathrm{mg} / \mathrm{m}^{2}$ intravenously q3 $\mathrm{wk}$, or doxorubicin $60 \mathrm{mg} / \mathrm{m}^{2}$ intravenously plus ifosfamide (range, $6-9 \mathrm{~g} / \mathrm{m}^{2}$ ) intravenously q3wk. PFS was the primary end point. Of 121 patients randomized, 88 had a translocation-related sarcoma confirmed by central review. PFS showed nonsignificant difference between the arms (stratified log rank test, $P=0.9573 ; \mathrm{HR}=0.86, P=0.6992$ ). There was no statistically significant difference in the survival curves. Response rate according to Response Evaluation Criteria in Solid Tumors (RECIST) was significantly higher in the chemotherapy arm, but response according to the Choi criteria showed fewer differences (45.9\% vs 37.3\%). ${ }^{17}$

Another study that has completed accrual is the Phase II (LMS-02) trial conducted by the French Sarcoma Group examining the activity of trabectedin plus doxorubicin versus doxorubicin alone in the first-line treatment of advanced nonoperable and/or metastatic leiomyosarcomas (uterine or soft tissue) and/or inoperable relapse. The primary end point in this study is the disease control rate (objective response rate and stable disease) per RECIST. Preliminary results suggest that the combination is tolerable, but final results are awaited. ${ }^{18}$

\section{Neoadjuvant setting}

In the neoadjuvant setting, a Phase II study in patients with advanced localized myxoid liposarcoma demonstrated encouraging activity. ${ }^{19}$ A Phase III study currently accruing is comparing the impact on disease-free survival of standard neoadjuvant chemotherapy (epirubicin and ifosfamide) with that of a neoadjuvant therapy tailored to the patient's histological subtype (NCT 01710176), eg, patients with myxoid liposarcoma with hypercellularity (round cell MPS) will receive trabectedin.

\section{Maintenance treatment and rechallenge}

Trabectedin does not have the same long-term toxicity as the cytotoxic agents commonly used to treat ASTS. For this reason, studies have addressed whether ongoing or 
maintenance treatment with trabectedin should be employed or whether trabectedin retains activity if patients are rechallenged on progression after a treatment break.

There is some evidence from a retrospective French study to support maintenance treatment with trabectedin. Patients treated in the French compassionate use program achieving a partial response or stable disease after six cycles of initial treatment with trabectedin $1.5 \mathrm{mg} / \mathrm{m}^{2}$ as a 24 -hour infusion every 3 weeks could proceed to maintenance treatment at their physician's discretion. Of 56 patients, 16 patients stopped treatment but 40 patients continued beyond six cycles for a median of 9 (range, 7-19). PFS and overall survival were significantly improved in the maintenance treatment group compared with the group that stopped after six cycles. ${ }^{20}$

This question has now been examined prospectively to determine whether trabectedin treatment beyond the sixth cycle in patients with ASTS demonstrating a response or stable disease should be continued. The study aim was to determine whether continual maintenance administration of trabectedin or pulsed therapy, in which patients stop after six cycles but are rechallenged at disease progression, was superior. The primary end point was PFS at 24 weeks post randomization. In the evaluable patients $(n=178)$, the rate of nonprogression after the initial six trabectedin cycles was 29.7\%. The median PFS after randomization was 7.2 months in the maintenance arm and 3.7 months in the pulsed arm $(P=0.05)$. Therefore, the median PFS was improved for those patients receiving the maintenance schedule when compared to a pulsed schedule. Additionally, the 12-month overall survival rate after randomization was $86 \%(62.4-95.3)$ and $74 \%$ (44.1-89.2) for the maintenance and pulsed arms, respectively. The authors concluded that trabectedin should therefore be given until progressive disease, intolerance, or patient choice to stop. ${ }^{21}$

\section{Combination therapy}

Preclinical data demonstrated synergism for doxorubicin and trabectedin in sarcoma cell lines, and the antitumor effects were additive when the two drugs were used in a murine xenograft sarcoma model. ${ }^{22,23}$ A Phase I study of doxorubicin plus trabectedin showing that administration of the two drugs concurrently was feasible and had an acceptable toxicity profile included some chemotherapy-naïve patients. The maximum tolerated dose was reached with doxorubicin $60 \mathrm{mg} / \mathrm{m}^{2}$ (with primary prophylactic growth factor support) and trabectedin $1.1 \mathrm{mg} / \mathrm{m}^{2} .{ }^{24}$ A Phase II trial, however, failed to show superior activity for the combination when compared with doxorubicin alone, and in fact the study was terminated early for futility. Therefore, despite the encouraging preclinical activity, the combination of trabectedin plus doxorubicin has not shown superiority in first-line ASTS patients. ${ }^{25}$ The reasons for the lack of activity are not entirely clear but may be due to the scheduling of the two agents. It is an area that warrants further attention.

\section{Differential trabectedin activity related to soft tissue sarcoma subtype}

There is a now a body of evidence in the form of clinical trials and anecdotal case reports of trabectedin activity in many different soft tissue sarcoma subtypes. From the early clinical trials with trabectedin, a higher proportion of objective responses and improved PFS were observed for patients with liposarcomas and leiomyosarcomas than for other softtissue sarcoma subtypes. ${ }^{26-28}$ Histologically, liposarcomas are classified into five different types: well-differentiated, myxoid, round cell, pleomorphic, and de-differentiated. Myxoid liposarcomas account for $40 \%$ of liposarcomas and almost $10 \%$ of all adult soft-tissue sarcomas and appear to be particularly sensitive to trabectedin. They can arise in any location and have an unusual pattern of metastasizing to soft tissues, bone, visceral surfaces, and the lung. They are characterized molecularly by specific chromosomal translocations: the more commonly occurring $\mathrm{t}(12 ; 16)(\mathrm{q} 13 ; \mathrm{p} 11)$ or the rarer $\mathrm{t}(12 ; 22)(\mathrm{q} 13 ; \mathrm{q} 12)$, resulting in the formation of FUS-CHOP or EWS-CHOP fusion proteins, respectively. CHOP is also known as DDIT3, and FUS also as TLS.

A compassionate use program was established in ASTS patients who had failed conventional chemotherapy to permit those who did not meet the eligibility criteria for trials and those in centers where trials were not active to receive trabectedin. In light of data from the earlier clinical trials and an impression in the compassionate-use program of a higher response rate and better tumor control for myxoid liposarcomas, a retrospective analysis of trabectedin in pretreated myxoid liposarcomas participating in the compassionate use program was undertaken. ${ }^{29}$ Fifty-one patients were included in the analysis recruited over a 5-year period (2001-2006) at five sarcoma centers worldwide. The overall objective response (complete response [CR] and partial response [PR] combined) was 51\% (95\% CI, 36-65) according to RECIST. Six patients had a minor response (MR), defined as a decrease in the sum of the longest diameter of target tumors not reaching criteria for an objective PR, and 14 patients had stable disease as best response. Therefore, overall tumor control 
was achieved in 46 out of 51 patients. It therefore seemed clear that patients with myxoid liposarcomas were particularly sensitive to trabectedin, and that the drug may, in part, be mediating its effect by interrupting the function of the characteristic translocations seen in this subtype.

The fusion proteins produced as a product of the translocations (FUS-CHOP or EWS-CHOP) act as abnormal transcription factors and are involved in the pathogenesis of the disease. Trabectedin displaces the oncogenic fusion protein from its target promoters, thereby halting its transcriptional activity. This ultimately induces adipogenic differentiation of myxoid liposarcoma cells. This phenomenon has been observed both in vitro and in vivo. ${ }^{9}$

FUS-CHOP presents 12 transcript variants that differ for the structure of the FUS-CHOP fusion genes. Exons 2, 3 , and 4 of the $C H O P$ gene are present in all fusion proteins except for type IV which lacks exon 2. The FUS component is much more variable in different groups. Type II contains exons from 1 to 5 , type 1 exons from 1 to 7 , and type III exons from 1 to 8 . The majority of patients $(70 \%)$ harbor the type I FUS-CHOP fusion gene, and a lower percentage presents with type I and III fusion genes. Other fusion types are extremely rare. ${ }^{9}$ The displacement of FUS-CHOP transcript variants (types I, II and III) from target promoters is similar but the kinetics of reattachment of the subtypes is different. Type III myxoid liposarcoma preclinical models appear to be less sensitive to the effects (antiangiogenic and differentiation) of trabectedin. In a retrospective analysis of myxoid liposarcomas receiving trabectedin by Grosso et al fluorescent in situ hybridization (FISH) analysis was conducted in 33 of the 51 patients and all 33 patients were found to have the $\mathrm{t}(12: 16)$ chromosomal translocation. In 12 of the 33 patients, frozen tissue permitted reverse transcriptase polymerized chain reaction (RT-PCR) to be conducted for the specific fusion transcript and demonstrated type II fusion transcript in eight patients: type 1 and IV in one patient; type II and IV in one patient; and type III in one patient; and type II and III in one patient. Interestingly, two of the patients with early progression had the presence of the rare type III transcript. ${ }^{29}$ While these data are intriguing, at this stage, they remain hypothesis-generating and the significance of the FUS-CHOP fusion transcripts on response requires to be tested prospectively.

\section{Challenge of assessing response to trabectedin}

A subgroup of 41 patients from the compassionate use program had centralized radiological review of imaging from baseline to best response, to progression, and to treatment end. Of 23 patients who achieved a confirmed PR or CR, only six of these responses had occurred by the first response assessment at a median of 8 weeks (interquartile range, 6-9). Of the other 17 patients at the first objective disease assessment, eight patients had a minor response at first assessment and nine had stable disease. Nondimensional tumor responses were observed, consisting of a decrease in tumor density on computerized tomography scan and/or a decrease in contrast enhancement on magnetic resonance imaging preceding their RECIST response. Additionally, some patients responded initially dimensionally and then nondimensionally. Median time to reach a dimensional decrease of the tumor amounting to an objective response was 3.6 months (interquartile range, 2.4-4.6). ${ }^{29}$ Interestingly, in the 17 patients who did not have a confirmed PR or CR by the first response assessment, early changes were visualized in tumor appearance on computerized tomography or magnetic resonance imaging scans. The characteristic feature was of a decrease in tumor density without substantial changes in tumor dimensions. Similar, early signs of tumor density decrease were seen in 12 of the 15 patients with stable disease as their best response confirmed on central imaging review. ${ }^{29}$

Therefore, the traditional method of using RECIST to assess response may not be the optimal means of assessing response with trabectedin. Clinicians should be aware when treating patients with trabectedin that a lack of a dimensional response does not necessarily mean that the tumor is failing to respond. For example, in the paper by Grosso et al a patient with a myxoid liposarcoma of the thigh who received four cycles of trabectedin demonstrated a marked decrease in contrast enhancement on imaging, without any tumor shrinkage. At surgery, the typical plexiform vascular pattern and the immature spindle cell neoplastic component had resolved and been replaced by sclerohyaline material in $70 \%$ of the sample. The rest of the sample showed adipocyte differentiation indicative of maturation. Equally, some patients do demonstrate a dimensional decrease in tumor size but these responses can be delayed and therefore the drug should not be stopped if there is evidence of disease stability but not of a RECIST response. The mechanisms underlying the different responses of tumors to this drug are not yet fully elucidated. However, there are several proposed mechanisms of action of trabectedin and these different mechanisms may result in different outcomes in terms of response. For example as mentioned previously, trabectedin may displace the oncogenic fusion transcript $F U S-C H O P$ from certain promoters within the tumor, resulting in changes in terminal adipogenesis and 
ultimately to maturation of the tumor along the adipogenic differentiation line. This mechanism of action is clearly different from its atypical alkylating action, which might be a more likely mechanism to lead to a dimensional decrease in tumor size. There may also be a mechanism of action of trabectedin affecting the tumor microenvironment together with a possible immunomodulatory and anti-inflammatory effect. ${ }^{30}$

In light of the unique and different way in which ASTS may respond to trabectedin, questions have been raised as to whether an alternative response assessment system should be used. For example, the Choi criteria, originally devised for gastrointestinal stromal tumors, interrogate a nondimensional aspect to response evaluating volume, density, vascularization, and other tumor features. ${ }^{31}$ Studies addressing the question of whether Choi criteria may be more useful than RECIST are ongoing, and include 1) the retrospective Therapeutic Response of Patients With Soft Tissue Sarcoma According to Choi Criteria (ProAcTyon) study, which is examining the prognostic value of Choi criteria compared with RECIST for assessment of trabectedin activity in patients with ASTS and 2) the Y-IMAGE study, which is a noninterventional multicenter prospective trial evaluating treatment outcome assessment methods used in routine clinical practice on patients with ASTS treated with trabectedin.

The design of trials in the second-line setting is made more difficult, as randomized Phase II studies comparing with the current gold standard are often employed and for ASTS there is no consensus about the optimal agent to use as second line. In addition to using Choi criteria, another alternative method of determining activity has been proposed, namely, the "growth modulation index" (GMI). This method relies on employing intrapatient comparison of successive TTP. GMI is defined as the ratio of the TTP with the second (or subsequent) line (TTPn) divided by the TTPn-1 with the $(n-1)$ th line of treatment. Central to this approach is the observation that TTP tends to become shorter with successive chemotherapeutic lines. The notion that the patient acts as his or her own internal control is appealing, potentially removing the influence of intrapatient heterogeneity on an overall trial result. However, the GMI method also has its challenges, principally the requirement to define an appropriate threshold for a "responder". It is also implicit that the TTPn-1 and TTP are known and accurately calculated to generate the ratio. The authors of a retrospective study defined the GMI ratio signifying activity as $>1.33$ as opposed to $>1.0$ in an attempt to eliminate chance fluctuations. They describe that this figure was chosen because response to successive chemotherapeutic agents tends to become shorter, and they therefore felt this was an acceptable cutoff. This study examined 279 patients with ASTS who had received trabectedin $1.5 \mathrm{mg} / \mathrm{m}^{2}$ (24-hour infusion every 3 weeks) in four phase II trials. The median TTPn was 2.8 months (range, 0.2-26.8) as compared with the median TTPn-1 of 4.0 months (0.3-79.5). Median GMI was $0.6(0.0-14.4)$. Overall, 177 patients $(63 \%)$ had a GMI $<1,21(8 \%)$ had a GMI equal to $1.0-1.33$, and $81(29 \%)$ had a GMI $>1.33$. These results correlated with the median overall survival in the three patient groups $(9.1,13.9$, and 23.8 months, respectively, $P=0.00005$ ). In addition, there was good concordance between the GMI and response rate $(P<0.0001)$ and PFS $(P<0.0001) .^{32}$

While this is an interesting retrospective analysis, this methodology would have to be tested prospectively to establish whether it is a suitable method of assessing response in ASTS in the second-line setting and whether it is superior or should be used in a complementary way with current assessment methods and trial end points. In tandem with treatment advances, it is imperative that novel approaches to assessing response are considered and tested. This is particularly relevant for a rare cancer such as sarcoma where a beneficial effect for one patient with a specific disease subtype may be obscured by the absence of a treatment effect for another patient with an alternative disease subtype.

\section{Safety considerations}

The first Phase II studies were conducted in 1999, and since then more than 1,000 patients have received trabectedin in the context of clinical trials, compassionate use programs, expanded access programs, or in standard clinical practice. A great deal is therefore known about the toxicities of this agent and how to manage patients safely on the drug. Trabectedin is now considered an agent that is generally well tolerated, and, as it does not have the limiting cumulative side effects of a chemotherapeutic agent, can be given to benefiting patients indefinitely. However, in the early trials, there were reports of many patients requiring dose delays and reductions; Grade 3-4 hematological toxicity with anemia, thrombocytopenia, and neutropenia was seen in a significant proportion of patients, as well as significant abnormalities in the liver transaminases reaching Grade 3-4 in approximately $50 \%$ of patients. Most seriously, treatment-related deaths were reported, which in some cases seemed to involve rhabdomyolysis. ${ }^{26,33}$ In the largest of these early studies, a statistical correlation was noted between liver dysfunction and the likelihood of experiencing Grade 3-4 toxicities. ${ }^{26}$

These early studies highlighted the link between liver function, drug clearance, and toxicity, in particular, 
myelosuppression and rhabdomyolysis. It became apparent that elevated transaminase levels with trabectedin are common and transient, tending to become less pronounced with repeated administration. However, a cholestatic picture of liver function derangement with an elevated bilirubin, alkaline phosphatase, and $\gamma$-glutamyl transpeptidase is associated with increased bone marrow toxicity due to poor drug clearance. It is now recommended that if liver function tests have not returned to near-normal by the time of the next administration of drug, a dose reduction is indicated. Failure to do this can result in a significant risk of increasingly severe myelosuppression.

A large retrospective pooled analysis conducted with 19 Phase II clinical trials in patients with advanced solid tumor $(n=1,132)$ treated with trabectedin as a 24-hour infusion every 3 weeks, a 3-hour infusion every 3 weeks, or a 3-hour infusion once weekly has provided a huge amount of safety data. ${ }^{34}$ The most common side effects were nausea, fatigue, and vomiting, although these were mild in most patients. Increases in transaminases that usually appeared early in the course of trabectedin exposure were reversible in most patients. A recurring pattern was seen with increased transaminases, typically reaching a peak between days 5 and 7 of each cycle and resolving to grade $\leq 1$ by day 15 without implications for the patient. Steroid pretreatment is an effective way of reducing the extent of hepatotoxicity, and steroids are now given routinely before trabectedin administration. ${ }^{35,36}$ Preclinical work has demonstrated that trabectedin causes necrosis of bile duct epithelial cells and that dexamethasone premedication in rats significantly reduces the hepatic levels of trabectedin in rats. ${ }^{37}$ This results in a reduction in liver damage possibly via the induction of cytochrome P450 enzymes. Importantly, this effect is achieved without compromising trabectedin's efficacy. Steroids can also reduce the likelihood of nausea or vomiting and therefore have a dual purpose. Neutropenia was also common, occurring in a third of patients, but treatment discontinuation for this reason was low (4.2\%) and febrile neutropenia was rare $(1.9 \%) .^{34}$

Rhabdomyolysis is another potentially serious adverse event. Elevations in creatinine kinase $(\mathrm{CK})$ are observed, but the incidence of muscle damage is low. Published data estimate the global incidence of rhabdomyolysis at $0.7 \%$ and the incidence of fatal cases at $0.3 \%$. No predictive factors have been identified to prospectively identify patients that may be at risk of this side effect, and therefore, to safely manage patients, serum $\mathrm{CK}$ monitoring is advisable. ${ }^{38}$
A recent retrospective study has also addressed the effect of age on the efficacy and safety of trabectedin, reporting that the response rate did not differ with age and PFS and overall survival rates were similar. The study reported a higher incidence of Grade 3-4 neutropenia (43.6\% vs 60.2\%) in patients $>60$ years and fatigue $(6.3 \%$ vs $14.4 \%)$. However, in 24 patients who were aged 70 years or over, no significant differences were seen in efficacy or safety outcomes. Therefore, trabectedin is a feasible treatment in all ages and an attractive choice for the palliative treatment of less fit elderly patients for whom chemotherapy may not be appropriate. ${ }^{39}$

\section{Conclusion}

Trabectedin is an active agent in ASTS, particularly in certain subgroups of the disease such as liposarcomas and leiomyosarcomas. The optimal schedule for administration as a single agent in advanced disease has been established, and a number of ongoing studies are addressing the question of using trabectedin earlier in the disease process, in the maintenance and rechallenge settings, and in combination with other therapeutic agents. Traditional methods of response assessment are prone to underestimating its activity with nondimensional alterations in tumors frequently seen. Therefore, caution must be exercised when decisions are being made regarding continuing or ceasing therapy. Where possible, a radiologist with understanding of the radiological changes seen with this agent can assist in accurately determining radiological benefit. Alternative or complementary assessment systems such as the Choi criteria may feature more commonly in assessing the response to agents such as trabectedin in the future, and further research into how best to assess disease response with imaging is warranted. As our understanding of the toxicities of the drug has increased, trabectedin can now be given safely with attention to hepatic dysfunction and the risk of rhabdomyolysis. Unlike chemotherapy, cumulative toxicity does not appear to be problematic, and benefiting patients can continue receiving trabectedin indefinitely. Future work should address those patients most likely to respond. Preliminary data in relation to the subtypes of the characteristic myxoid liposarcoma translocation relating to response are interesting. With the knowledge of histopathological subtypes and the discovery of characteristic molecular subtypes, trials are endeavoring to determine which patients are most sensitive to novel agents although the relative rarity of sarcomas in general continues to make the conduct of the traditional clinical trial problematic. International collaborative working is critical if trials are to be conducted in a timely fashion and in discrete sarcoma subtypes. 


\section{Disclosure}

The authors report no conflicts of interest in this work.

\section{References}

1. Leahy M, Garcia Del Muro X, Reichardt P, et al. Chemotherapy treatment patterns and clinical outcomes in patients with metastatic soft tissue sarcoma. The SArcoma treatment and Burden of Illness in North America and Europe (SABINE) study. Ann Oncol. 2012;23(10):2763-2770.

2. Van Glabbeke M, van Oosterom AT, Oosterhuis JW, et al. Prognostic factors for the outcome of chemotherapy in advanced soft tissue sarcoma: an analysis of 2,185 patients treated with anthracyclinecontaining first-line regimens - a European Organization for Research and Treatment of Cancer Soft Tissue and Bone Sarcoma Group Study. J Clin Oncol. 1999;17(1):150-157.

3. van Oosterom AT, Mouridsen HT, Nielsen OS, et al; EORTC Soft Tissue and Bone Sarcoma Group. Results of randomised studies of the EORTC Soft Tissue and Bone Sarcoma Group (STBSG) with two different ifosfamide regimens in first- and second-line chemotherapy in advanced soft tissue sarcoma patients. Eur $J$ Cancer. 2002;38(18):2397-2406.

4. Judson I, Verweij J, Gelderblom H, et al; European Organisation and Treatment of Cancer Soft Tissue and Bone Sarcoma Group. Doxorubicin alone versus intensified doxorubicin plus ifosfamide for first-line treatment of advanced or metastatic soft-tissue sarcoma: a randomised controlled phase 3 trial. Lancet Oncol. 2014;15(4):415-423.

5. Jin S, Gorfajn B, Faircloth G, Scotto KW. Ecteinascidin 743, a transcription-targeted chemotherapeutic that inhibits MDR1 activation. Proc Natl Acad Sci U S A. 2000;97(12):6775-6779.

6. Minuzzo M, Marchini S, Broggini M, Faircloth G, D'Incalci M, Mantovani R. Interference of transcriptional activation by the antineoplastic drug ecteinascidin-743. Proc Natl Acad Sci U S A. 2000;97(12):6780-6784.

7. Feuerhahn S, Giraudon C, Martínez-Díez M, et al. XPF-dependent DNA breaks and RNA polymerase II arrest induced by antitumor DNA interstrand crosslinking-mimetic alkaloids. Chem Biol. 2011;18(8):988-999.

8. Forni C, Minuzzo M, Virdis E, et al. Trabectedin (ET-743) promotes differentiation in myxoid liposarcoma tumors. Mol Cancer Ther. 2009;8(2):449-457.

9. Di Giandomenico S, Frapolli R, Bello E, et al. Mode of action of trabectedin in myxoid liposarcomas. Oncogene. Epub 2013 November 11.

10. Bueren-Calabuig JA, Giraudon C, Galmarini CM, Egly JM, Gago F. Temperature-induced melting of double-stranded DNA in the absence and presence of covalently bonded antitumour drugs: insight from molecular dynamics simulations. Nucleic Acids Res. 2011;39(18):8248-8257.

11. Aune GJ, Takagi K, Sordet O, et al. Von Hippel-Lindau-coupled and transcription-coupled nucleotide excision repair-dependent degradation of RNA polymerase II in response to trabectedin. Clin Cancer Res. 2008;14(20):6449-6455.

12. D'Incalci M, Badri N, Galmarini CM, Allavena P. Trabectedin, a drug acting on both cancer cells and the tumour microenvironment. Br J Cancer. 2014;111(4):646-650.

13. Atmaca H, Bozkurt E, Uzunoglu S, Uslu R, Karaca B. A diverse induction of apoptosis by trabectedin in MCF-7 (HER2-/ER+) and MDA-MB-453 (HER2+/ER-) breast cancer cells. Toxicol Lett. 2013; 221(2):128-136.

14. Demetri GD, Chawla SP, von Mehren M, et al. Efficacy and safety of trabectedin in patients with advanced or metastatic liposarcoma or leiomyosarcoma after failure of prior anthracyclines and ifosfamide: results of a randomized phase II study of two different schedules. J Clin Oncol. 2009;27(25):4188-4196.

15. Van Glabbeke M, Verweij J, Judson I, Nielsen OS. Progression-free rate as the principal end-point for phase II trials in soft-tissue sarcomas. Eur J Cancer. 2002;38(4):543-549.
16. Janssen Research \& Development, LLC. A study of trabectedin or dacarbazine for the treatment of patients with advanced liposarcoma or leiomyosarcoma. Available from: http://clinicaltrials.gov/show/ NCT01343277. NLM identifier: NCT01343277. Accessed November 4, 2014.

17. Blay JY, Leahy MG, Nguyen BB, et al. Randomised phase III trial of trabectedin versus doxorubicin-based chemotherapy as first-line therapy in translocation-related sarcomas. Eur $J$ Cancer. 2014;50(6):1137-1147.

18. Pautier P, Floquet A, Cupissol D, et al. LMS-02: a phase II single arm multicenter study of doxorubicin in combination with trabectedin as a first line treatment for advanced uterine leiomyosarcoma (U-LMS) and soft tissue LMS (ST-LMS): first results in patients with U-LMS. J Clin Oncol. 2013;31(suppl; abstr 10505).

19. Gronchi A, Bui BN, Bonvalot S, et al. Phase II clinical trial of neoadjuvant trabectedin in patients with advanced localized myxoid liposarcoma. Ann Oncol. 2012;23(3):771-776.

20. Saada E, Rahal C, Ray-Coquard I, et al. Rechallenge with trabectedin in patients with locally advanced or metastatic soft tissue sarcoma following drug holiday: the experience of the French Sarcoma Group (FSG). J Clin Oncol. 2012;30(suppl; abstr 10062).

21. Le Cesne A, Blay JY, Ryckewaert T, et al. Results of the prospective T-DIS randomized phase II trial comparing interruption versus continuation of trabectedin after six cycles of treatment in patients (pts) with advanced soft tissue sarcomas (ASTS). J Clin Oncol. 2014;32(5s suppl; abst 10523).

22. Meco D, Colombo T, Ubezio P, et al. Effective combination of ET-743 and doxorubicin in sarcoma: preclinical studies. Cancer Chemother Pharmacol. 2003;52(2):131-138.

23. Takahashi N, Li WW, Banerjee D, Scotto KW, Bertino JR. Sequencedependent enhancement of cytotoxicity produced by ecteinascidin 743 (ET-743) with doxorubicin or paclitaxel in soft tissue sarcoma cells. Clin Cancer Res. 2001;7(10):3251-3257.

24. Blay JY, von Mehren M, Samuels BL, et al. Phase I combination study of trabectedin and doxorubicin in patients with soft-tissue sarcoma. Clin Cancer Res. 2008;14(20):6656-6662.

25. Martin-Broto J, Lopez-Pousa A, Garcia del Muro X, et al. Randomised phase II trial of doxorubicin versus trabectedin plus doxorubicin in first line treatment of patients with advanced non-resectable or metastatic soft tissue sarcomas: a Spanish Group for Sarcoma Research (GEIS) study. Eur Cancer Congr. (17 ECCO-38 ESMO-32 ESTRO); 2013 Sep 27-Oct 1; Amsterdam. Publicado en: Eur J Cancer. 2013;49(suppl 2):S875. [Abstr.3800].

26. Garcia-Carbonero R, Supko JG, Manola J, et al. Phase II and pharmacokinetic study of ecteinascidin 743 in patients with progressive sarcomas of soft tissues refractory to chemotherapy. J Clin Oncol. 2004; 22(8):1480-1490.

27. Le Cesne A, Blay JY, Judson I, et al. Phase II study of ET-743 in advanced soft tissue sarcomas: a European Organisation for the Research and Treatment of Cancer (EORTC) soft tissue and bone sarcoma group trial. J Clin Oncol. 2005;23(3):576-584.

28. Garcia-Carbonero R, Supko JG, Maki RG, et al. Ecteinascidin-743 (ET-743) for chemotherapy-naive patients with advanced soft tissue sarcomas: multicenter phase II and pharmacokinetic study. J Clin Oncol. 2005;23(24):5484-5492.

29. Grosso F, Jones RL, Demetri GD, et al. Efficacy of trabectedin (ecteinascidin-743) in advanced pretreated myxoid liposarcomas: a retrospective study. Lancet Oncol. 2007;8(7):595-602.

30. D'Incalci M, Galmarini CM. A review of trabectedin (ET-743): a unique mechanism of action. Mol Cancer Ther. 2010;9(8): 2157-2163.

31. Choi H, Charnsangavej C, Faria SC, et al. Correlation of computed tomography and positron emission tomography in patients with metastatic gastrointestinal stromal tumor treated at a single institution with imatinib mesylate: proposal of new computed tomography response criteria. J Clin Oncol. 2007;25(13):1753-1759. 
32. Penel N, Demetri GD, Blay JY, et al. Growth modulation index as metric of clinical benefit assessment among advanced soft tissue sarcoma patients receiving trabectedin as a salvage therapy. Ann Oncol. 2013;24(2):537-542.

33. Yovine A, Riofrio M, Blay JY, et al. Phase II study of ecteinascidin-743 in advanced pretreated soft tissue sarcoma patients. J Clin Oncol. 2004;22(5):890-899.

34. Le Cesne A, Yovine A, Blay JY, et al. A retrospective pooled analysis of trabectedin safety in 1,132 patients with solid tumors treated in phase II clinical trials. Invest New Drugs. 2012;30(3):1193-1202.

35. Paz-Ares L, López-Pousa A, Poveda A, et al. Trabectedin in pre-treated patients with advanced or metastatic soft tissue sarcoma: a phase II study evaluating co-treatment with dexamethasone. Invest New Drugs. 2012;30(2):729-740.
36. Grosso F, Dileo P, Sanfilippo R, et al. Steroid premedication markedly reduces liver and bone marrow toxicity of trabectedin in advanced sarcoma. Eur J Cancer. 2006;42(10):1484-1490.

37. Donald S, Verschoyle RD, Greaves P, et al. Complete protection by highdose dexamethasone against the hepatotoxicity of the novel antitumor drug yondelis (ET-743) in the rat. Cancer Res. 2003;63(18):5902-5908.

38. Grosso F, D'Incalci M, Cartoafa M, et al. A comprehensive safety analysis confirms rhabdomyolysis as an uncommon adverse reaction in patients treated with trabectedin. Cancer Chemother Pharmacol. 2012;69(6):1557-1565.

39. Cesne AL, Judson I, Maki R, et al. Trabectedin is a feasible treatment for soft tissue sarcoma patients regardless of patient age: a retrospective pooled analysis of five phase II trials. Br J Cancer. 2013; 109(7):1717-1724.

\section{Publish your work in this journal}

Therapeutics and Clinical Risk Management is an international, peerreviewed journal of clinical therapeutics and risk management, focusing on concise rapid reporting of clinical studies in all therapeutic areas, outcomes, safety, and programs for the effective, safe, and sustained use of medicines. This journal is indexed on PubMed Central, CAS,
EMBase, Scopus and the Elsevier Bibliographic databases. The manuscript management system is completely online and includes a very quick and fair peer-review system, which is all easy to use. Visit http://www.dovepress.com/testimonials.php to read real quotes from published authors.

Submit your manuscript here: http://www.dovepress.com/therapeutics-and-clinical-risk-management-journal 